\title{
Polarization Analysis in Mössbauer Reflectometry with Synchrotron Mössbauer Source
}

\author{
Marina Andreeva ${ }^{1, *(\mathbb{D}}$, Roman Baulin $\left.{ }^{1} \mathbb{(}\right)$, Aleksandr Chumakov ${ }^{2,3} \mathbb{D}$, Tatiyana Kiseleva ${ }^{1}$ and \\ Rudolf Rüffer ${ }^{2}$ \\ 1 Faculty of Physics, M.V. Lomonosov Moscow State University, 119991 Moscow, Russia; \\ baulin.roman@physics.msu.ru (R.B.); kiseleva.tyu@gmail.com (T.K.) \\ 2 ESRF-The European Synchrotron, CS 40220, 38043 Grenoble CEDEX 9, France; chumakov@esrf.fr (A.C.); \\ rueffer@esrf.fr (R.R.) \\ 3 National Research Centre "Kurchatov Institute", Pl. Kurchatova 1, 123182 Moscow, Russia \\ * Correspondence: Mandreeva1@yandex.ru; Tel.: +07-903-712-0837
}

Received: 1 October 2018; Accepted: 2 January 2019; Published: 8 January 2019

\begin{abstract}
Polarization selection of the reflected radiation has been employed in Mössbauer reflectivity measurements with a synchrotron Mössbauer source (SMS). The polarization of resonantly scattered radiation differs from the polarization of an incident wave so the Mössbauer reflectivity contains a scattering component with $90^{\circ}$ rotated polarization relative to the $\pi$-polarization of the SMS for some hyperfine transitions. We have shown that the selection of this rotated $\pi \rightarrow \sigma$ component from total reflectivity gives an unusual angular dependence of reflectivity characterized by a peak near the critical angle of the total external reflection. In the case of collinear antiferromagnetic interlayer ordering, the "magnetic" maxima on the reflectivity angular curve are formed practically only by radiation with this rotated polarization. The first experiment on Mössbauer reflectivity with a selection of the rotated polarization discovers the predicted peak near the critical angle. The measurement of the rotated $\pi \rightarrow \sigma$ polarization component in Mössbauer reflectivity spectra excludes the interference with non-resonant electronic scattering and simplifies the spectrum shape near the critical angle allowing for an improved data interpretation in the case of poorly resolved spectra. It is shown that the selected component of Mössbauer reflectivity with rotated polarization is characterized by enhanced surface sensitivity, determined by the "squared standing waves" depth dependence. Therefore, the new approach has interesting perspectives for investigations of surfaces, ultrathin layers and multilayers having complicated magnetic structures.
\end{abstract}

Keywords: X-ray reflectivity; Mössbauer spectroscopy; magnetic multilayers; standing waves

\section{Introduction}

Interaction of light with magnetized media is characterized by specific polarization dependences. That is true as well for X-ray radiation. Modern sources of synchrotron radiation produce X-rays of any desired polarization and polarization-dependent absorption or scattering near the X-ray absorption edges (XMCD—X-ray magnetic circular dichroism, XMLD—X-ray magnetic linear dichroism, XRMR-X-ray resonant magnetic reflectivity) [1-7] have become extremely effective methods of magnetic investigation. For non-resonant $X$-ray scattering, the polarization analysis has been applied for separation of the spin and orbital magnetic moments and magnetic structure investigations [8-13]. Polarization analysis has been used for the observation of the X-ray Faraday and Kerr effects with soft X-rays [14-22].

For Mössbauer radiation, the splitting of the nuclear levels by hyperfine interactions means simultaneously the energy separation of the absorbed or reemitted quanta by their polarization 
states. Theoretical description of the elliptical polarization for different hyperfine transitions was done long ago [23]. The polarization dependences of Mössbauer absorption and Faraday rotation in thick samples were theoretically developed and experimentally proved in the excellent paper of Blume and Kistner [24]. In conventional Mössbauer spectroscopy, the radioactive sources emit an un-polarized single line radiation and the polarization state of different absorption lines has not been of special interest. However, the polarization state of various absorption lines reveals itself in the ratio of their intensity. In this way, the spectrum shape characterizes the direction of the sample magnetization [25].

The nuclear resonance ("Mössbauer") experiments with synchrotron radiation have been started by using the specific way of the nuclear response registration: by measuring the time evolution of the delayed nuclear decay after prompt SR (synchrotron radiation) pulse [26,27]. In this time-domain approach, the hyperfine splitting of nuclear levels leads to the quantum beats in the nuclear decay, and the polarization state of various hyperfine components becomes essential: it determines the result of their interference. The waves with orthogonal polarizations do not interfere. To be more precise, the coherent addition of the waves with orthogonal polarizations does not give the interference term in the resulting intensity [28]. For example, when magnetization of the sample is parallel to the beam, the four hyperfine transitions excited by $\sigma$-polarized SR give just one quantum beat frequency in nuclear decay [29]. However, the measurements of the scattered radiation with polarization selection immediately show all possible frequencies of the quantum beats, because the linear polarization, resulting from coherent addition of the two circular polarization, rotates with the time delay. That was splendidly demonstrated in the papers of Siddons et al. [30,31].

In time-domain Mössbauer spectroscopy, the polarization analysis of the scattered radiation has been found to be very helpful for the separation of the delayed nuclear scattering from huge prompt electronic scattering at the initial moment of pulse excitation. The electronic scattering does not change the polarization state of the incident radiation whereas the scattering at hyperfine nuclear sublevels gives the $90^{\circ}$-rotated polarization component. Therefore, the polarization selection of this rotated component supplies very efficient suppression of the non-resonant prompt response, allowing nuclear decay monitoring from the very short delay times (after $\sim 1$ ns of their excitation) [30,32,33].

Recent developments of the nuclear resonance beamlines make it possible now the energy-domain Mössbauer spectroscopy with SR. In particular, the nuclear ${ }^{57} \mathrm{FeBO}_{3}$ monochromator (synchrotron Mössbauer source-SMS) has been installed at the ID18 beamline of the European synchrotron (ESRF) and at the BL11XU beamline of SPring-8 [34-37]. The key point of the SMS is the pure nuclear (111) or (333) reflection (forbidden for electronic diffraction) of the iron borate ${ }^{57} \mathrm{FeBO}_{3}$ crystal, which provides a single-line purely $\pi$-polarized $14.4 \mathrm{keV}$ radiation within the energy bandwidth of $8 \mathrm{neV}$. The crystal should be heated at a specific temperature close to the Neél point of $348.35 \mathrm{~K}$. In comparison with the laboratory experiments, the application of the $\pi$-polarized beam results in new features of Mössbauer spectra measured with SMS in absorption or reflection geometry [38]. Use of a diamond phase plate in addition to the ${ }^{57} \mathrm{FeBO}_{3}$ monochromator at the BL11XU beamline gives the new possibilities to perform measurements in forward and grazing-incidence geometries with various (linear, circular, elliptical) polarization states of radiation [39].

Polarization analysis of the resonantly reflected radiation by magnetic multilyers has not been used before. For comparison in the nonresonant magnetic X-ray scattering the polarization analysis was effectively used for magnetic structure investigations. In polarized neutron reflectivity, the spin-flip analysis provides very valuable information. Therefore, we suppose that polarization analysis in Mössbauer reflectivity should be useful.

In this work, the first results demonstrating the peculiarities of the nuclear resonant reflectivity (Mössbauer reflectivity) with SMS supplemented by the selection of the component with rotated $\pi \rightarrow \sigma$ polarization are presented. We show the differences in the Mössbauer reflectivity angular dependencies and Mössbauer reflectivity spectra measured without and with selection of the $\pi \rightarrow \sigma$ component, and we explain new features of the $\pi \rightarrow \sigma$ reflectivity using the X-ray standing wave 
approach. The practical significance of this new development for the complicated spectra treatment or depth-resolved investigations is also discussed.

\section{Theory}

The amplitudes of the nuclear resonant scattering in the forward direction, including the change of the polarization $v \rightarrow v^{\prime}$, in the case of the dipole nuclear resonant transitions and in the presence of hyperfine splitting of the nuclear levels have the following expression $[40,41]$ :

$$
f_{j}^{n u c l}\left(\omega, v \rightarrow v^{\prime}\right)=-\frac{1}{2 \lambda} \sigma_{r e s} \frac{2 L+1}{2 I_{e}+1} f_{j}^{L M} \sum_{m_{e}, m_{g}} \frac{\frac{\Gamma_{j}}{2}\left|\left\langle I_{g} m_{g} L \Delta m \mid I_{e} m_{e}\right\rangle\right|^{2}}{\hbar \omega-E_{j R}\left(m_{e}, m_{g}\right)+\frac{i \Gamma_{j}}{2}}\left[\vec{h}_{j \Delta m} \circ \vec{h}_{j \Delta m}^{*}\right]_{v \rightarrow v^{\prime}}
$$

where $\hbar \omega$ is the photon energy, $\lambda$ is the corresponding radiation wavelength. For $14.4 \mathrm{kev} \mathrm{M} 1$ transition in ${ }^{57} \mathrm{Fe} L=1, I_{e}=3 / 2, I_{g}=1 / 2, m_{e}, m_{g}$ are the magnetic quantum numbers, $\left\langle I_{g} m_{g} L \Delta m \mid I_{e} m_{e}\right\rangle$ are the Clebsch-Gordan coefficients, $\sigma_{\text {res }}=2.56 \times 10^{-4} \mathrm{~nm}^{2}$ is the resonant cross-section, $\lambda=0.086 \mathrm{~nm}$, $j$ numerate the kinds of the hyperfine splitting (i.e., different multiplets in Mössbauer spectrum), $f_{j}^{L M}$ is the Lamb-Mössbauer factor, $\hat{h}_{\Delta m}$ in (1) are the spherical unit vectors in the hyperfine field principal axis $\vec{h}_{x}, \vec{h}_{y}, \vec{h}_{z}$ :

$$
\vec{h}_{ \pm 1}=\mp i \frac{\vec{h}_{x} \pm i \vec{h}_{y}}{\sqrt{2}}, \vec{h}_{0}=i \vec{h}_{z}
$$

and the sign $\circ$ designates the outer product of these spherical unit vectors.

Considering grazing incidence and specifying the orientation of the hyperfine magnetic field $\mathbf{B}_{h f}$ by polar $\beta$ and azimuth $\gamma$ angles, the angular dependences of the nuclear resonant scattering amplitude for different hyperfine transitions $\Delta m=m_{e}-m_{g}= \pm 1,0$ can be presented as matrices in $\sigma-, \pi$-polarization basis vectors:

$$
\begin{gathered}
f_{\Delta m=0}^{n u c l, \perp} \propto\left(\begin{array}{cc}
\sin ^{2} \beta \cos ^{2} \gamma & -\sin \beta \cos \beta \cos \gamma \\
-\sin \beta \cos \beta \cos \gamma & \cos ^{2} \beta
\end{array}\right) \\
f_{\Delta m= \pm 1}^{n u c l, \perp} \propto \frac{1}{2}\left(\begin{array}{cc}
\sin ^{2} \gamma+\cos ^{2} \gamma \cos ^{2} \beta & (\cos \beta \cos \gamma \mp i \sin \gamma) \sin \beta \\
(\cos \beta \cos \gamma \pm i \sin \gamma) \sin \beta & \sin ^{2} \beta
\end{array}\right)
\end{gathered}
$$

(we determine $\beta$ relative the sample normal and choose $\gamma=0^{\circ}$ for the direction in surface plane perpendicular to the beam) The non-diagonal matrix elements of the nuclear resonant scattering mean the appearance of the $90^{\circ}$ rotated polarization components in the scattered radiation.

For magnetic dipole (M1) nuclear transition (as it takes place for $14.4 \mathrm{kev}$ transition in ${ }^{57} \mathrm{Fe}$ ), the matrices in (3), (4) should be considered for the magnetic field of radiation. Therefore, the vector-column of the magnetic field of radiation for the $\pi$-polarized incident radiation from SMS is represented as $\left(\begin{array}{l}1 \\ 0\end{array}\right)$, and the first columns in (3), (4) describe the angular dependences and polarization properties of the amplitudes of the nuclear resonant scattering $f_{\Delta m}^{n u c l}$ in our case. It follows from (3), (4) that for $\Delta m=0$ transitions the rotated $\pi \rightarrow \sigma$ polarization component appears in the scattering intensity only if $\mathbf{B}_{h f}$ has a non-zero projection on the normal to the surface. For $\Delta m= \pm 1$ transitions the rotated $\pi \rightarrow \sigma$ polarization component is created if $\mathbf{B}_{h f}$ lies in the surface plane $\left(\beta=90^{\circ}\right.$, but not for $\gamma=0^{\circ}$ and maximal for $\beta=90^{\circ}, \gamma=90^{\circ}$ ). Later we consider such planar magnetic structures, typical for thin films.

Mössbauer absorption spectra are determined by the imaginary part of the scattering amplitude (1) according to the optical theorem: $\sigma(\omega)=2 \lambda \sum_{j} \operatorname{Im} f_{j}^{n u c l}(\omega)(\sigma(\omega)$ is the absorption cross section). Mössbauer reflectivity spectra are not similar to the Mössbauer absorption spectra, but are distorted by the interference with the electronic scattering, and their shape strongly depends on the grazing 
angle. In the simplest case of semi-infinite mirror Mössbauer reflectivity spectra are calculated with the Fresnel formula, in which the refractive index is simply connected with the scattering amplitudes by electrons $f^{e l}$ and nuclei $f^{n u c l}$ (diagonal components in (1)): $n=1+\frac{\lambda^{2}}{2 \pi} \rho\left(f^{e l}+f^{n u c l}\right)$ ( $\rho$ is the density of scatters) [42]. In the case of multilyers the multiple interference of waves reflected by all boundaries and subjected to the polarization transformation essentially complicates the theory. The total algorithm of the Mössbauer reflectivity calculations based on the $4 \times 4$-propagation matrices is rather lengthy and is not presented here. It has been described in many papers [43-45]. In the kinematical approximation, which is applicable at the larger grazing angles than the critical angle, the shape of the Mössbauer reflectivity spectra can be qualitatively described by $\left|\sum_{j} f_{j}^{n u c l}(\omega)\right|^{2}$ for thin single layer and we can use (1) for evaluation of the ratio of lines in Mössbauer reflectivity spectra. For several layers or periodic structures, the phase shifts for the scattered waves should be taken into account. For instance, for the structure with antiferromagnetic interlayer coupling between ${ }^{57}$ Fe layers the angular and polarization dependencies for the spectrum lines are described by other polarization matrices than (3), (4) (see Ref. [38]).

We start with the model calculations of the Mössbauer reflectivity angular curves and Mössbauer reflectivity spectra at several grazing angles using our computer code RESPC (available from the ESRF website [46]). The essential point is that the calculations have been performed with selection of the reflected radiation by the polarization state and the result is shown in Figure 1 . The $\pi \rightarrow \pi$ reflectivity is drawn by thicker blue lines, $\pi \rightarrow \sigma$ reflectivity is drawn by thinner red lines with symbols.

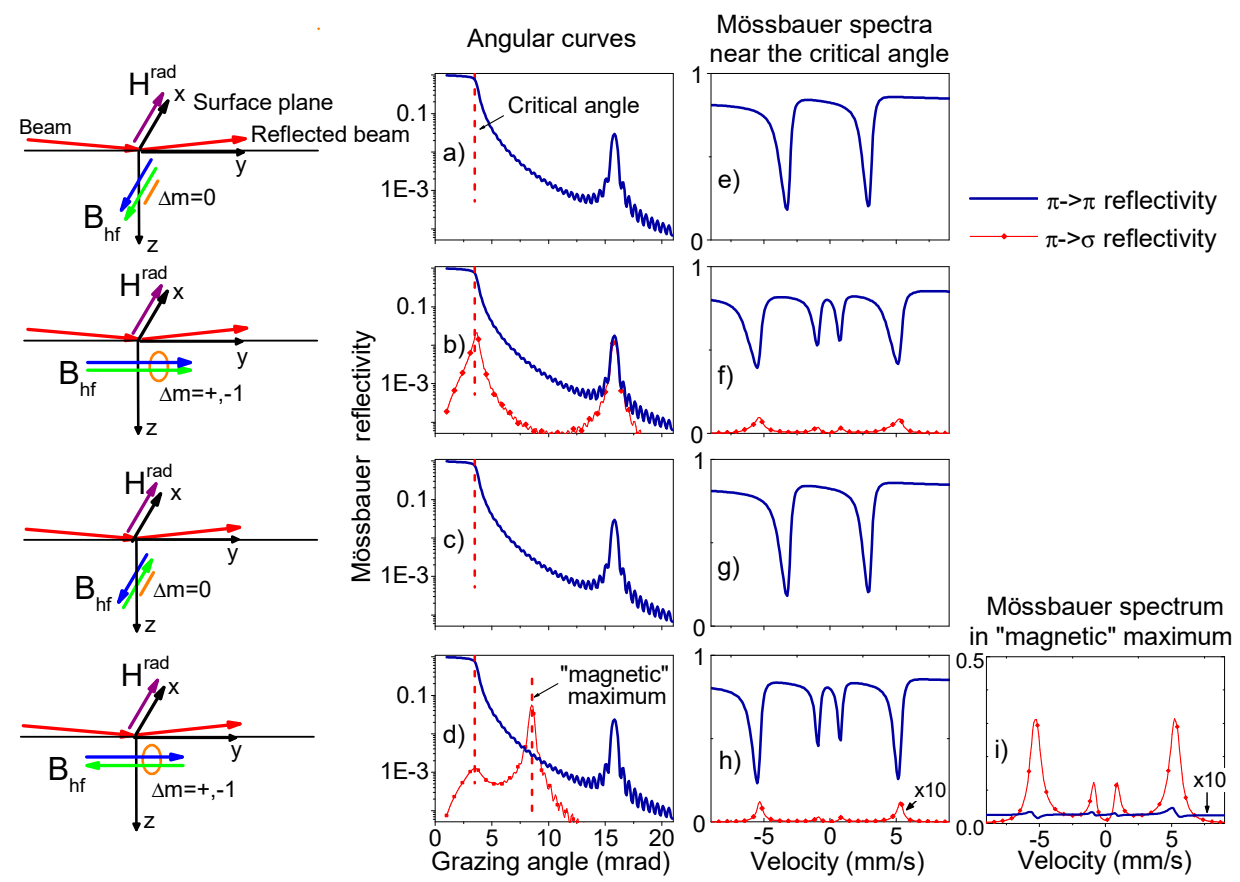

Figure 1. Model calculation of the angular dependencies of the Mössbauer reflectivity (a-d) and Mössbauer reflectivity spectra at the critical angle (e-h) and in the "magnetic" maximum (i) for $\pi$-polarized SMS radiation and for different cases of the ferromagnetic and antiferromagnetic coupling between adjacent ${ }^{57} \mathrm{Fe}$ layers, schematically drawn on the left.

In the energy-domain, the angular dependence of the Mössbauer reflectivity can be calculated either for a selected energy in the resonant spectrum range, or as the integral over the entire Mössbauer reflectivity spectra at each grazing angle $\theta$. Figure $1 \mathrm{a}-\mathrm{d}$ show the results obtained by the integrated mode, this mode corresponds to the experimental procedure with SMS. Calculations have been done for the $\left[{ }^{57} \mathrm{Fe}(0.8 \mathrm{~nm}) / \mathrm{Cr}(2 \mathrm{~nm})\right]_{30}$ multilayer, in ${ }^{57} \mathrm{Fe}$ layers we assume the presence of the hyperfine magnetic field of $\mathrm{B}_{\mathrm{hf}}=33 \mathrm{~T}$ with $\Delta \mathrm{B}_{\mathrm{hf}}=1 \mathrm{~T}$ distribution. We have considered the two 
magnetization directions relative to the radiation beam and the two types of the interlayer coupling between adjacent ${ }^{57} \mathrm{Fe}$ layers (ferromagnetic or antiferromagnetic), schematically shown by the blue and green arrows in the left column of Figure 1. Note that $14.4 \mathrm{keV}$ Mössbauer transition is of magnetic dipole $\mathrm{M} 1$ type, so the magnetic field of radiation $\mathrm{H}^{\text {rad }}$ interacts with ${ }^{57} \mathrm{Fe}$ nuclei. In the case of the $\pi$-polarized radiation from SMS the radiation field vector $\mathrm{H}^{\text {rad }}$ lies in the sample surface. The hyperfine nuclear transitions $(\Delta \mathrm{m}=0, \Delta \mathrm{m}= \pm 1)$ allowed for $\pi$-polarized radiation are also indicated by orange lines in these sketches.

The results of these simple calculations show some unexpected features, not reported in previous experimental studies. The shapes of the angular curves of the Mössbauer $\pi \rightarrow \pi$ and $\pi \rightarrow \sigma$ reflectivity are found very different. For Mössbauer $\pi \rightarrow \pi$ reflectivity the angular dependences $I^{\pi \rightarrow \pi}(\theta)$ behave as usual X-ray reflectivity (they tend to 1 when $\theta$ ). This behavior follows directly from usual Fresnel law, and it had been observed in the first paper devoted to Mössbauer reflectivity [42].

On the contrary, the angular dependencies $I^{\pi \rightarrow \sigma}(\theta)$ of the Mössbauer $\pi \rightarrow \sigma$ reflectivity have a sharp peak at the critical angle of the total external reflection and approach zero when $\theta \rightarrow 0$, as it seen in Figure 1b,d.

For the antiferromagnetic interlayer coupling, the magnetic period of the structure is twice larger than the chemical period. In this case, the additional Bragg peak ("magnetic" maximum) appears in the Mössbauer reflectivity angular dependence (see Figure 1d). Our calculations show that only the radiation with rotated $\pi \rightarrow \sigma$ polarization contributes to this peak (provided that there are no asymmetrically canted $\mathrm{B}_{\mathrm{hf}}$ in the adjacent ${ }^{57} \mathrm{Fe}$ layers). Accordingly, the Mössbauer spectrum at the magnetic maximum is determined practically only by $\pi \rightarrow \sigma$ scattering (see Figure 1i). The rotated polarization component appears in the reflected signal only when the hyperfine field $\mathrm{B}_{\mathrm{hf}}$ has a finite projection on the beam direction, i.e., when the excitation of the resonant $\Delta m= \pm 1$ transitions leads to the reemission of radiation with some part of the circular polarization. Accordingly, the Mössbauer reflectivity spectra with rotated polarization show not six but only four lines corresponding to the $\Delta m= \pm 1$ transitions as it takes place in Figure $1 \mathrm{f}, \mathrm{h}, \mathrm{i}$. In the other cases, there is no reflectivity with rotated polarization at all.

Note that the discovered behavior of the angular dependencies of the Mössbauer $\pi \rightarrow \sigma$ reflectivity resembles much the angular dependence of the delayed nuclear resonant reflectivity measured in time-domain experiments [47]. For the delayed reflectivity, the vanishing of the delayed photons at zero grazing angle also can be explained by the Fresnel law: when $\theta \rightarrow 0$ the reflectivity for all energies in Mössbauer spectrum approaches unity, entirely losing its energy dependence. Accordingly, the intensity of the delayed radiation, determined by the Fourier transform of the energy dependent reflectivity amplitude, approaches zero. The physical explanation of the peak on the delayed reflectivity curve has been suggested in $[48,49]$, and it is based on the phenomena of the X-ray standing waves. It has been shown that these waves, created by the prompt SR pulse, are responsible for the excitation of resonant nuclei. The X-ray standing wave explanation works in the case of the Mössbauer $\pi \rightarrow \sigma$ reflectivity in the energy-domain as well.

The basis for such consideration is the expression, obtained in [49], for the reflectivity from an ultrathin layer $r^{\prime}$ in the case when this ultrathin layer is placed at some depth $z$ in a multilayer:

$$
r^{\prime}(z)=T(z) T^{\prime}(z)\left(1+R^{\text {below }}(z)\right)^{2} r
$$

where

$$
r=i \frac{\pi \chi}{\lambda \sin \theta} d
$$

is the reflectivity from an separated ultrathin layer of thickness $d, \theta$ is the grazing angle,

$$
\chi=\frac{\lambda^{2}}{\pi} \rho f
$$


is the susceptibility of this layer, $\rho$ is the volume density of the scattering centers and $f$ the amplitude of scattering in forward direction. $R^{\text {below }}(z)$ corresponds to the reflectivity amplitude from the part of the multilayer below the ultrathin layer, and functions $T(z) T^{\prime}(z)$ describe the transformations of the transmitted and outgoing waves during multiple reflections at all boundaries in the upper part of the multilayer:

$$
\begin{aligned}
T(z) T^{\prime}(z) & =e^{2 i\left(\varphi_{1}+\varphi_{2}+\ldots+\varphi_{j-1}\right)} \\
& \times \frac{\left(1-r_{1}^{2}\right)\left(1-r_{2}^{2}\right) \ldots\left(1-r_{j-1}^{2}\right)}{\left(1+r_{1} R_{2} e^{2 i \varphi_{1}}\right)^{2}\left(1+r_{2} R_{3} e^{2 i \varphi_{2}}\right)^{2} \ldots\left(1+r_{j-1} R_{j} e^{2 i \varphi_{j-1}}\right)^{2}}
\end{aligned}
$$

In (8) $r_{j}$ and $R_{j}$ are the Fresnel and multiple reflectivity amplitudes respectively at each boundary above the ultrathin layer,

$$
\varphi_{j}=\frac{2 \pi}{\lambda} d_{j} \sqrt{\sin ^{2} \theta+\chi_{j}}
$$

is the phase shift for the waves during their transmission through each layer $j$. It is easy to see that

$$
T(z) T^{\prime}(z)\left(1+R^{\text {below }}(z)\right)^{2}=E^{2}(z)
$$

where $E(z)$ is the total amplitude of the radiation field at depth $z$ and $|E(z)|^{2}$ is no other than a standing wave. Assuming that $f, \chi$ and $r$ in (7), (6) are the matrices in the case of the anisotropic scattering like (3), (4), the following expression can be written for the off-diagonal component of the reflectivity (supposing sufficiently small) from the whole multilayer in the case of $\pi$-polarization of the incident radiation:

$$
I^{\pi \rightarrow \sigma}(\theta, \omega)=\frac{\lambda^{2}}{\sin ^{2} \theta}\left|\int \rho_{r e s}(z) f_{r e s}^{\pi \rightarrow \sigma}(z, \omega) E_{\pi}^{2}(\theta, z, \omega) d z\right|^{2} .
$$

This component appears only due to the nuclear resonance contribution to the susceptibility and $f_{\text {res }}^{\pi \rightarrow \sigma}$ is determined by the angular dependencies in (3), (4). The accurate derivation of (11) is presented in Ref. [50], and its application to the X-ray resonant magnetic reflectivity has been demonstrated in Ref. [51].

The presented expression (11) has important consequences on the features of the Mössbauer reflectivity with rotated polarization. Firstly, it explains perfectly the peak near the critical angle on the angular curve for the rotated $\pi \rightarrow \sigma$ reflectivity, because it contains the full radiation field $E(\theta, z, \omega)$ in the 4th power ("squared standing wave"). It is well known that the angular dependence of the secondary radiation, excited by X-ray standing waves, is characterized by the peak at the total reflection angle [52-54]. Secondly, the secondary radiation emission depends on the normal standing waves $|E(\theta, z)|^{2}$. The $\left|E^{2}(\theta, z, \omega)\right|^{2}$ factor in (11) ("squared standing wave") supplies the enhanced depth contrast comparing with $|E(\theta, z, \omega)|^{2}$, which is illustrated by Figure 2 . Thirdly, the expression (11) essentially simplifies and fastens the calculations of the reflectivity with rotated polarization from anisotropic multilayers. It means that if the dichroic contribution to the scattering is small, the standing waves $E(\theta, z, \omega)$ at depth $z$ of the sample can be calculated using the ordinal scalar reflectivity theory, and the "dichroic" response $I^{\pi \rightarrow \sigma}(\theta, \omega)$ is calculated by simple summation the scattering amplitudes $f_{\text {res }}^{\pi \rightarrow \sigma}(z, \omega)$ from different depth with proper "weights" (the generalized kinematical approximation). If the nuclear resonance scattering is strong enough, the radiation field $E_{\pi}(\theta, z, \omega)$ varies across the resonant spectrum (as seen in Figure 3b); however, in most cases these variations of $E_{\pi}(\theta, z, \omega)$ as a function of $\omega$ can be neglected especially if we measure the NRR (Nuclear Resonance Refelctivity) curves by integrating over Mössbauer reflectivity spectra. In this case the calculations become extremely fast.

The enhanced depth selectivity of the Mössbauer $\pi \rightarrow \sigma$ reflectivity spectra compared with the ordinal Mössbauer reflectivity spectra is illustrated by Figure 3. In order to distinguish the thin top layer $\left(1.5 \mathrm{~nm}{ }^{57} \mathrm{Fe}\right)$ from the bottom one, we assumed for the top layer the hyperfine magnetic field $\mathrm{B}_{\mathrm{hf}}=28 \mathrm{~T}$, and for the deeper ${ }^{57}$ Fe layer $\left(3 \mathrm{~nm}\right.$ thickness) $\mathrm{B}_{\mathrm{hf}}=30 \mathrm{~T}$. The calculated Mössbauer 
reflectivity spectra at several grazing angles near the critical one without and with $\pi \rightarrow \sigma$ polarization selection clearly show that the $\pi \rightarrow \sigma$ reflectivity spectra contain more intense contribution from the $1.5 \mathrm{~nm}$ top layer than the Mössbauer reflectivity spectra without polarization selection.

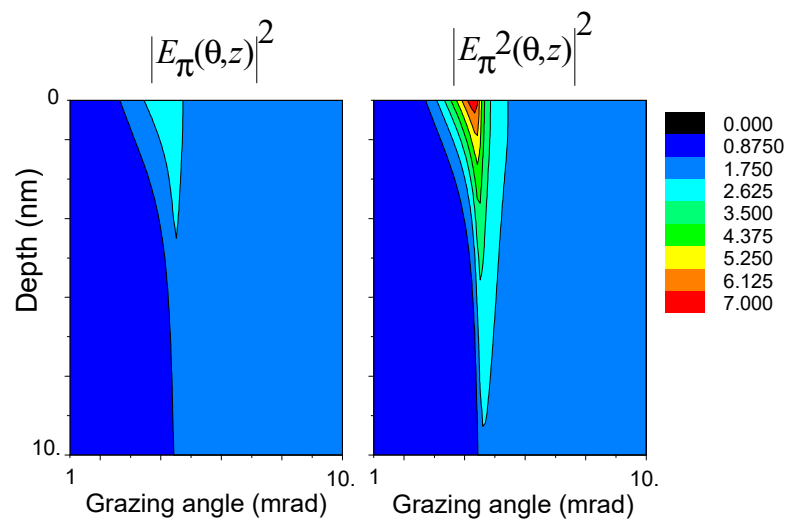

Figure 2. Comparison of the angular-depth dependence of the standing wave $\left|E_{\pi}(\theta, z, \omega)\right|^{2}$ (left panel) and squared standing wave $\left|E_{\pi}^{2}(\theta, z, \omega)\right|^{2}$ (right panel), calculated for an iron mirror neglecting the nuclear resonant contribution to the scattering.

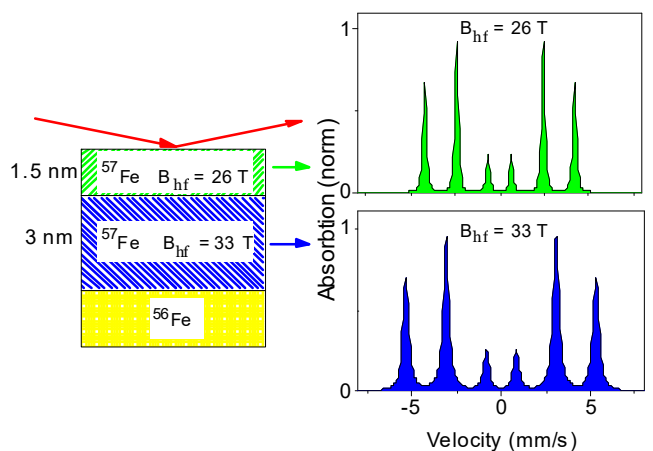

(a)

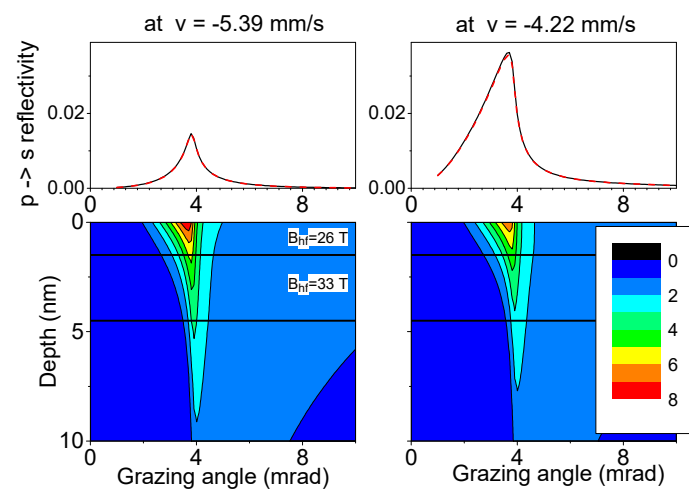

(b)

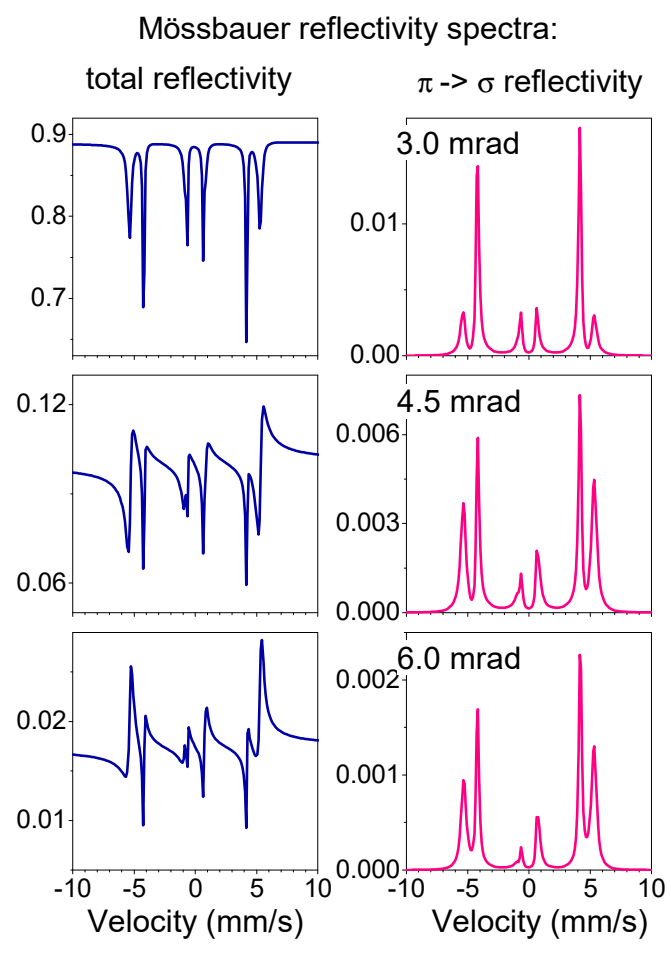

(c)

Figure 3. (a) The used in calculations model: two thin layers with slightly different $B_{\text {hf }}$ (their resonant spectra are on the left), directed along the beam. (b, bottom panels) The angular-depth distribution of the squared standing waves $\left|E_{\pi}^{2}(\theta, z, \omega)\right|^{2}$, calculated for two indicated velocities (v) points in the resonant spectrum, the solid horizontal lines show the layers boundaries, (b, upper panels) the angular curves for $\pi \rightarrow \sigma$ reflectivity, corresponding to this energy points. The solid and dashed lines (nearly indistinguishable) show the results, obtained by Equation (1) and by the exact calculations, performed with the program REFSPC [46]. (c) The Mössbauer reflectivity spectra without the polarization selection (left column) and for $\pi \rightarrow \sigma$ reflectivity (right column), calculated for three indicated grazing angles in vicinity of the critical angle. 
The validity of the generalized kinematical approximation (11) is also checked by calculations presented in Figure 3b. The angular curves in Figure 3b are calculated in two ways: by the formula (11) and by the exact calculations performed with the program pack REFSPC [46] (solid and dashed lines respectively). Both results coincide. However, in order to achieve this practically full coincidence, the nuclear resonant susceptibility was decreased by a factor of 5 relative to the value for the $\alpha$-iron films fully enriched by ${ }^{57} \mathrm{Fe}$ isotope. Such decreased nuclear resonance susceptibility is typical for real thin films with lower enrichment and broad distribution of the hyperfine magnetic fields. Thereby, the direct comparison between the kinematical approximation (11) and the exact calculations shows the excellent agreement, provided that the resonant contribution to the susceptibility is not too large.

The next advantage of the measurements with the selection of polarization is illustrated by Figure 4. It shows that the selection of polarization leads to the decreasing number of lines in the Mössbaur reflectivity spectra. This can be very useful for the interpretation of the poorly resolved complicated spectra. The experimental Mössbaur reflectivity spectrum from Ref. [55], presented on the top of Figure 4, can be fitted by at least three different models with completely different field orientation and distribution of the hyperfine magnetic field. The calculated $\pi \rightarrow \sigma$ reflectivity spectra are quite different for these three cases. Therefore, the true picture of the hyperfine field distribution and orientation can be recovered if one performs the polarization analysis of the reflected radiation.

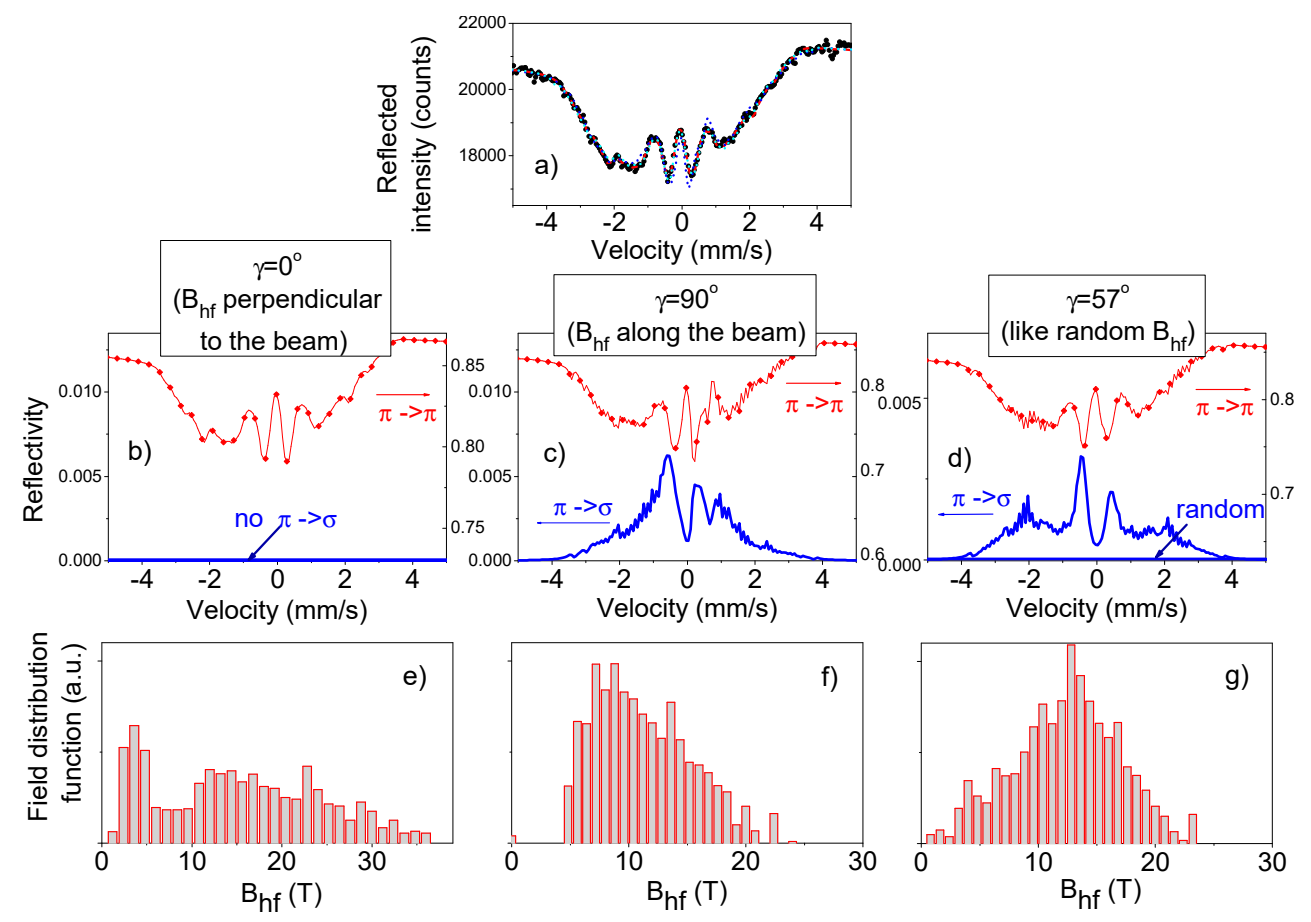

Figure 4. (a) Mössbauer reflectivity spectrum from Ref. [55], measured without polarization selection for the $\left[{ }^{57} \mathrm{Fe}(0.12 \mathrm{~nm}) / \mathrm{Cr}(1.05 \mathrm{~nm})\right] \times 30$ sample in remanence. Symbols are the experimental data, three solid (practically undistinguishable) lines are the results of the fit with three different orientations of the hyperfine magnetic field $\mathrm{B}_{\mathrm{hf}}$ : for $\mathrm{B}_{\mathrm{hf}}$ in the surface plane and perpendicular to the beam (azimuthal angle $\left.\gamma=0^{\circ}\right), B_{h f}$ parallel to the beam $\left(\gamma=90^{\circ}\right)$, and $B_{h f}$ in the surface plane with $\gamma=57^{\circ}$ (for this angle the spectrum without polarization analysis is the same as in the case of the random in space orientations of $\left.B_{h f}\right)$. (b-d) Theoretical Mössbauer reflectivity spectra for these models with the selection of polarization: solid red lines with symbols are the results for the reflectivity with nonrotated $(\pi \rightarrow \pi)$ polarization, solid blue lines are those for the rotated $(\pi \rightarrow \sigma)$ polarization component in the reflectivity. (e-g) The corresponding different field distributions $\mathrm{P}\left(\mathrm{B}_{\mathrm{hf}}\right)$ for these three cases, giving the same result of the fit to the experimental spectrum shown in (a). 


\section{Experimental Results and Discussion}

The experiment has been performed at the Nuclear Resonance beamline [56] ID18 of the ESRF. Figure 5 shows the experimental setup. The storage ring was operated in multi-bunch mode with a nominal current of $200 \mathrm{~mA}$. The energy bandwidth of radiation was first reduced down to $2.1 \mathrm{eV}$ by the high-heat-load monochromator [57] adjusted to the $14.4125 \mathrm{keV}$ energy of the nuclear resonant transition of the ${ }^{57} \mathrm{Fe}$ isotope. Then $\mathrm{X}$-rays were collimated by the compound refractive lenses down to the angular divergence of a few $\mu \mathrm{rad}$. The high-resolution monochromator decreases the energy bandwidth of the beam further to $\sim 15 \mathrm{meV}$. Final monochromatization down to the energy bandwidth of $\sim 8 \mathrm{neV}$ was achieved with the pure nuclear (111) reflection of the ${ }^{57} \mathrm{FeBO}_{3}$ crystal, and the sweep through the energy range of a Mössbauer resonant spectrum (about $\pm 0.5 \mu \mathrm{eV}$ ) was achieved using the Doppler velocity scan. Radiation from the SMS was focused vertically using the compound refractive lenses down to the beam spot of $50 \mu \mathrm{m}$. The intensity of the X-ray beam incident on the sample was about $10^{4}$ photons/s. More details on the design of the SMS can be found in Ref [37].

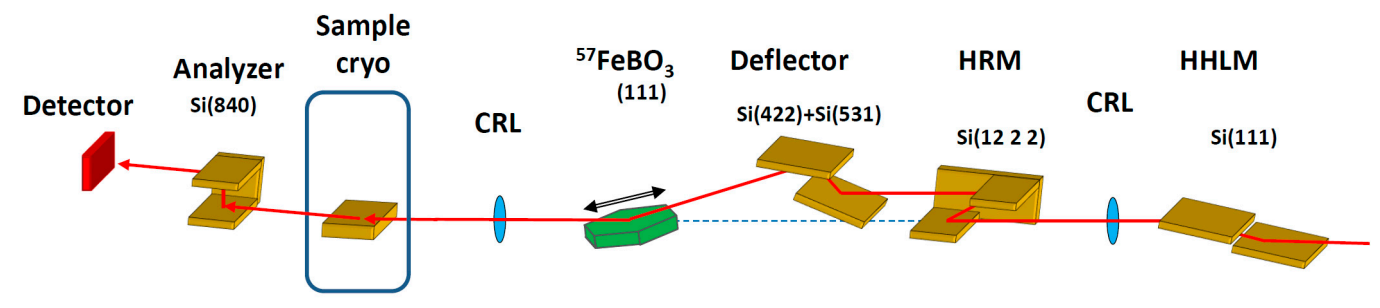

Figure 5. Experimental set-up for measurements of the Mössbauer reflectivity with the selection of radiation with the rotated $(\pi \rightarrow \sigma)$ polarization. HHLM-high-heat-load monochromator; CRL—compound refractive lenses, HRM—high-resolution monochromator.

The selection of the radiation with the rotated $(\pi \rightarrow \sigma)$ polarization was performed by silicon channel-cut crystal with two asymmetric $\operatorname{Si}(840)$ reflections with the Bragg angle $\theta_{\mathrm{B}}=45.1^{\circ}$. According to theoretical calculations, the channel-cut should suppress the $\pi$-polarized radiation by about five orders of magnitude, while transmitting about $70 \%$ of the $\int$-polarized radiation with the angular acceptance of about 2.6 arc sec (FWHM).

We studied several $\left[{ }^{57} \mathrm{Fe} / \mathrm{Cr}\right]_{30}$ samples. The samples were grown with the Katun-C molecular beam epitaxy facility, equipped by 5 thermal evaporators at the Institute of Metal Physics (Ekaterinburg, Russia). The growth was performed under the UHV (Ultra-high vacuum) regime $\left(5 \times 10^{-10} \mathrm{mbar}\right)$. During the buffer layer deposition, the $\mathrm{Al}_{2} \mathrm{O}_{3}$ substrate temperature was gradually decreased from $300{ }^{\circ} \mathrm{C}$ down to $180{ }^{\circ} \mathrm{C}$. The typical deposition rate of $\mathrm{Cr}$ and ${ }^{57} \mathrm{Fe}$ layers was about of $0.15 \mathrm{~nm} / \mathrm{min}$. The details on the preparations and characterization of the samples can be found elsewhere [38]. The samples were mounted in the cassette holder of the He-exchange gas superconducting cryo-magnetic system. The studies were performed at helium temperature (4.0 K).

The angular scan with the polarization analyzer, performed for all used samples in order to catch the $\pi \rightarrow \sigma$ reflected radiation, demonstrated the extremely broadened divergence of the reflected signal as it is shown in Figure 6 for one of the sample. In essence, the samples were composed of many nano-crystallites, essentially misoriented relative to each other. Consequently, the radiation reflected by the sample consisted of many spikes, spreading over the angular range of about 200 arc sec as seen in Figure 6. For such a divergent reflected beam, the throughput of the $\mathrm{Si}(840)$ channel-cut analyzer for the $\sigma$-polarized radiation was only about $4 \%$. Therefore, the measurements of the reflectivity with the rotated $\pi \rightarrow \sigma$ polarization were essentially complicated by the nano-islands structure of the studied samples. Later we get the GISAXS (Grazing-incidence small-angle X-ray scattering) pattern from this sample which shows the cluster-layered structure of the whole film [58]. 


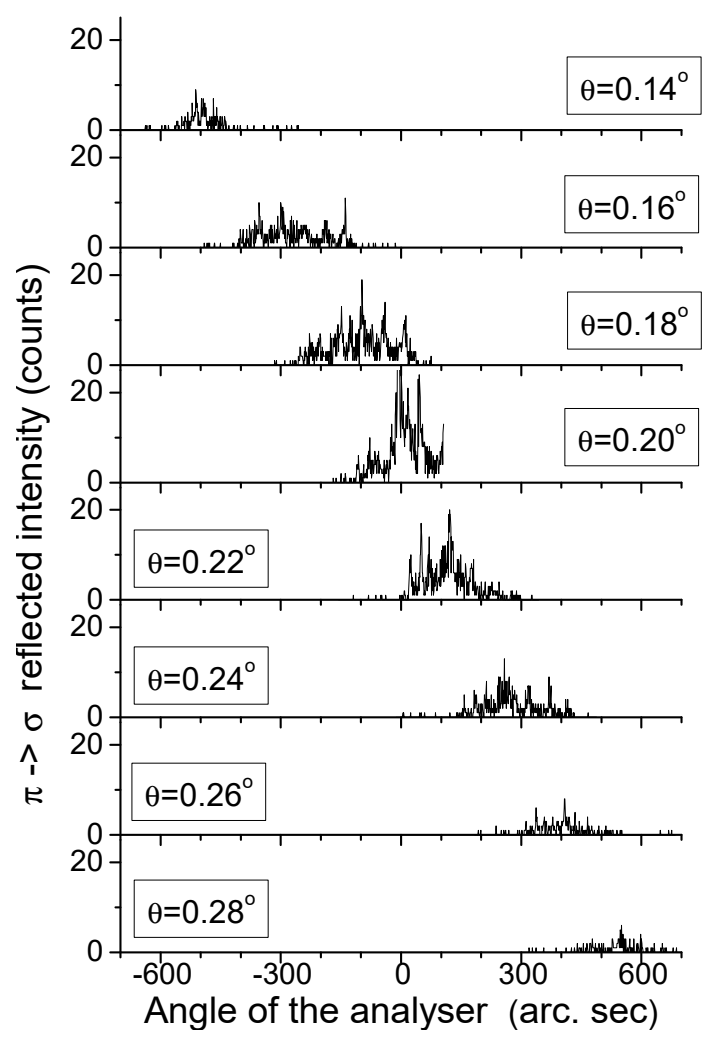

Figure 6. Angular distributions of the radiation reflected with $\pi \rightarrow \sigma$ rotation of the polarization from the $\left[{ }^{57} \mathrm{Fe}(0.8 \mathrm{~nm}) / \mathrm{Cr}(1.05 \mathrm{~nm})\right]_{30}$ sample measured with the $\mathrm{Si}(840)$ channel-cut analyzer for various grazing angles $\theta$.

With the resulting very small count rate, the measurements at the weak "magnetic" maxima for the samples with antiferromagnetic interlayer coupling were not feasible, and we decided to concentrate on measurements near the critical angle. In order to ensure the ferromagnetic alignment of the magnetization in the ${ }^{57} \mathrm{Fe}$ layers and increase the Mössbauer dichroic reflectivity, the external magnetic field of $5 \mathrm{~T}$ was applied along the beam direction.

In order to increase the count rate, the temperature of the ${ }^{57} \mathrm{FeBO}^{3}$ crystal for this particular measurement was lowered by few degrees. This sacrifices the energy resolution of the SMS to $\sim 230 \mathrm{neV}(\sim 5 \mathrm{~mm} / \mathrm{s})$, which is not important for the angular dependency measurements, but increases the intensity of the SMS by an order of magnitude [37]. The nuclear resonance reflectivity with the rotated polarization shown in Figure 7 was obtained for each grazing angle $\theta$ by integrating over the angular distributions measured with the $\mathrm{Si}(840)$ analyzer and drawn in Figure 6.

The predicted peak near the critical angle for the Mössbauer $\pi \rightarrow \sigma$ reflectivity is clearly seen in Figure 7. The origin of this peak is already explained in the previous section. The angular dependence of the Mössbauer reflectivity measured without the polarization selection shows the plateau below the critical angle. Note that the signal values for two curves in Figure 7 are not possible to compare because they are measured with different incident intensities from ${ }^{57} \mathrm{FeBO}_{3}$ nuclear monochromator.

Mössbauer $\pi \rightarrow \sigma$ reflectivity spectra were measured at the two grazing angels, $\theta=0.19^{\circ}$ and $\theta=0.22^{\circ}$, in vicinity of the critical angle. They are shown in Figure 8. In spite of the limited statistics, caused by the essential loss of the intensity due to the nano-islands structure of the samples and narrow angular reception by ideal Si crystal-analyzer, we can recognize in these data some important features. The resonant lines are presented in these spectra as peaks, as predicted by the theory (see Figure 1f,h,g). The spectrum measured without polarization analysis is also presented in Figure 8 for comparison. The lines in this spectrum have a dispersive-like shape and they are essentially broadened, as it should be at the angles near the critical angle [46]. Note, that in spite of the higher statistical accuracy 
of the spectrum measured without the polarization analysis, its smeared shape makes the correct interpretation more difficult. Thus, the spectra of reflectivity with rotated polarization should be more suited for the right data analysis, provided that better statistics will be reached by optimization of the experiment.

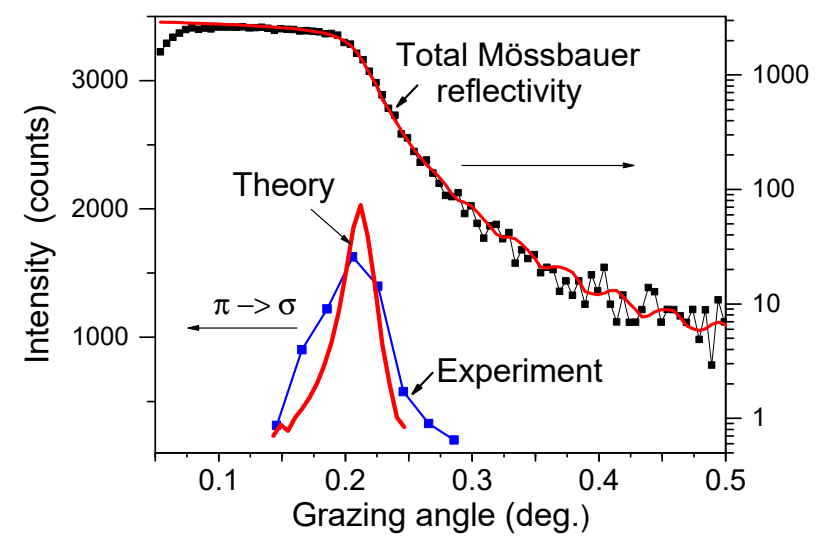

Figure 7. Angular dependencies of the Mössbauer reflectivity measured without selection of polarization and with the selection of the rotated $\pi \rightarrow \sigma$ component in reflectivity, obtained as the integral over scans, presented in Figure 6. The lines with symbols show the experimental data. The red solid lines show the results of theoretical calculations.

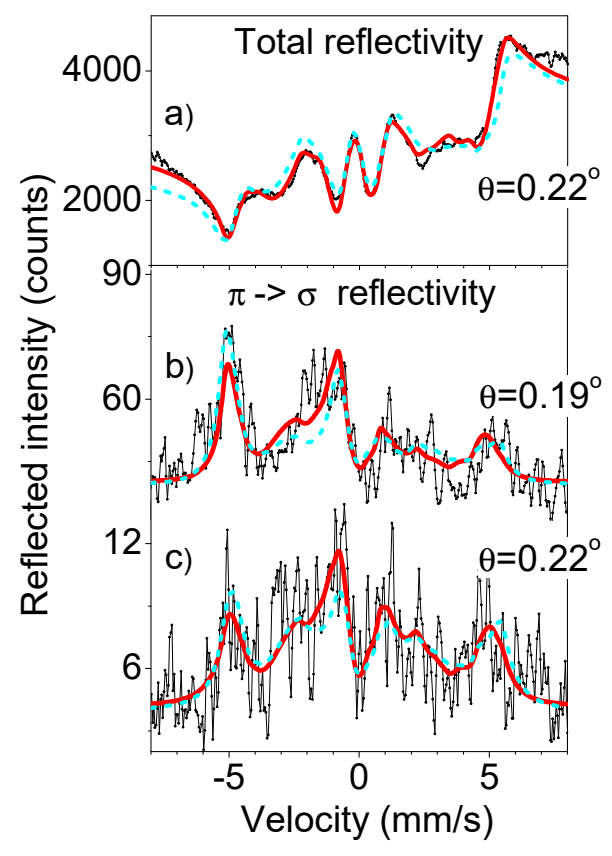

Figure 8. Mössbauer reflectivity spectra measured from $\left[{ }^{57} \mathrm{Fe}(0.8 \mathrm{~nm}) / \mathrm{Cr}(1.05 \mathrm{~nm})\right]_{30}$ multilayer without polarization analysis at the critical angle (a) and with selection of the rotated polarization component at the two angles in the vicinity of the critical angle $(\mathbf{b}, \mathbf{c})$. The solid lines with symbols are the experimental data. The red sold and green dashed lines are the fits discussed in the text.

The fit of the $\pi \rightarrow \sigma$ reflectivity spectra is not really reliable due to insufficient statistics, we merely wanted to show what treatment should be performed with better experimental data and demonstrate the surface sensitivity of the method. So, the shapes of the spectra in Figure 8 can be more or less reproduced by the fit performed with the REFSPC program package [46]. All three spectra are treated within the same model. We have used seven hyperfine fields $B_{h f}{ }^{(i)}, i=1,2, \ldots, 7$, and the fitted parameters and distribution probabilities of the fields $P\left(B_{h f}\right)$ are shown in Figure $9 \mathrm{a}$. 


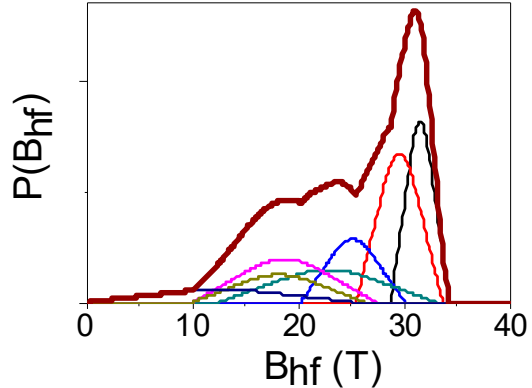

(a)

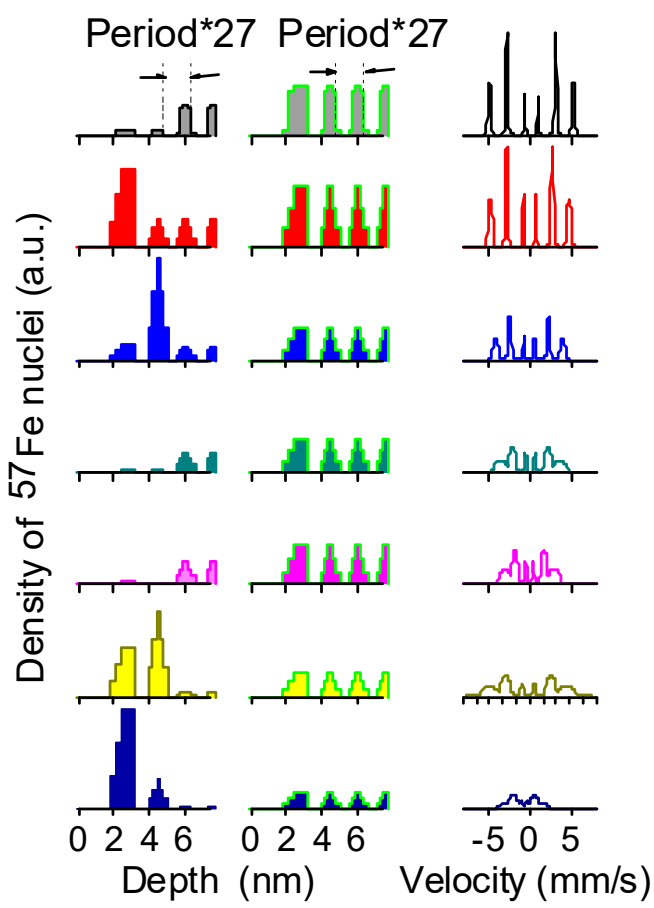

(b)

Figure 9. (a) Hyperfine field distribution, obtained by the fit of the spectra in Figure 8. (b) Depth distribution of the each kind of $B_{h f}{ }^{(i)}(i=1,2, \ldots, 7)$ in several top ${ }^{57}$ Fe layers (the period, repeating 27 times in the deeper part of the multilayer, is marked, the last period contacted with the buffer layer is also different from the main periodic part but is not shown here), obtained by the fit (left column); the initially suggested homogeneous depth distribution of $B_{h f}{ }^{(i)}$ in all ${ }^{57} \mathrm{Fe}$ layers (middle column), the calculated reflectivity spectra for this distribution are presented in Figure 8 by the dashed green lines. In the right column, the resonant spectra corresponding to every $\mathrm{B}_{\mathrm{hf}}{ }^{(\mathrm{i})}$ are shown.

The depth profiles obtained for each field $\mathrm{B}_{\mathrm{hf}}{ }^{(\mathrm{i})}$ are presented in the left column of Figure $9 \mathrm{~b}$. We can see that the hyperfine fields in the top two ${ }^{57} \mathrm{Fe}$ layers are significantly different from the fields in the deeper layers: on average, in the top layers the hyperfine splitting is smaller, and the fraction of the low field contributions is larger. Assuming the same field distribution in the top layers as in the whole periodic part (shown in Figure 9b, middle column), we obtain the theoretical spectra, presented by dashed green lines in Figure 8 . They reveal worse correspondence with the experimental spectra. These calculations confirm the enhanced depth selectivity of the spectra of reflectivity with rotated $\pi \rightarrow \sigma$ polarization.

Other unexpected features of the measured spectra were noticed. We were surprised by the asymmetry and by the difference in shapes of the $\pi \rightarrow \sigma$ reflectivity spectra measured at the two close angels: $\theta=0.19^{\circ}$ and $\theta=0.22^{\circ}$ (compare the spectra in Figure $8 b, c$ ). At the beginning we supposed that these effects are caused by a small contribution of the reflectivity with the non-rotated polarization. But the model calculations did not confirm such assumption. Finally, the effect was explained by the refraction effect.

For illustration of this effect we use a simple model. Calculations have been performed for the $10 \mathrm{~nm}{ }^{57} \mathrm{Fe}$ layer where two hyperfine fields $\mathrm{B}_{\mathrm{hf}}=28 \mathrm{~T}$ and $30 \mathrm{~T}$ take place, the resonant spectra for these two fields are drown in Figure 10b. The angular dependencies of the Mössbauer $\pi \rightarrow \sigma$ reflectivity, calculated for the photon energies corresponding to two outer lines in these spectra reveal the different angular positions of the critical angle peaks which is clearly seen in Figure 10c. This shift of the peak positions explains the asymmetry of the rotated $\pi \rightarrow \sigma$ polarization component in Mössbauer reflectivity spectra. 


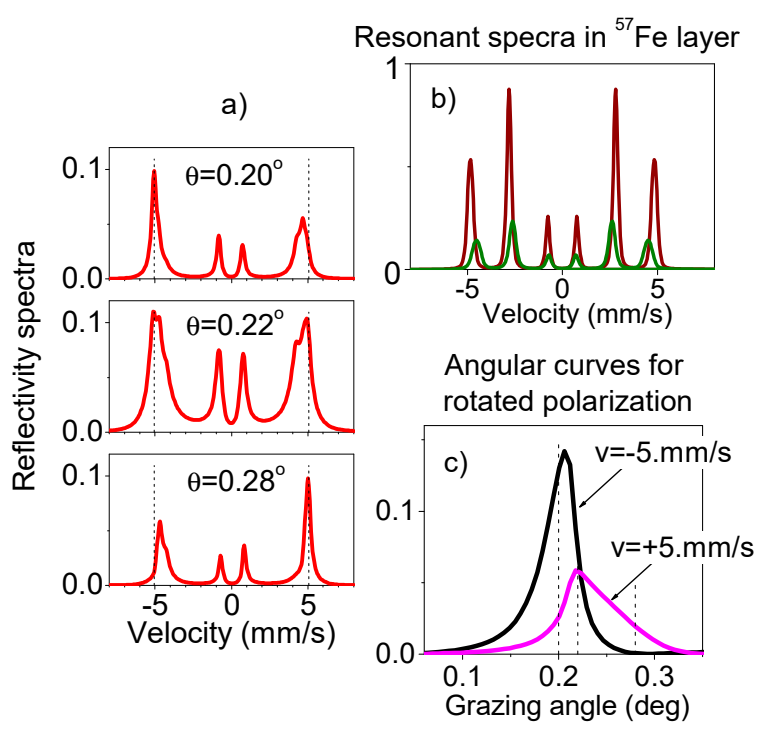

Figure 10. (a) Model calculations of the rotated $\pi \rightarrow \sigma$ polarization component in Mössbauer reflectivity spectra. Dashed vertical lines mark the energies, for which the angular dependencies of Mössbauer reflectivity in (c) are calculated. (b) The resonant spectra for the $\mathrm{B}_{\mathrm{hf}}=30 \mathrm{~T}$ and $28 \mathrm{~T}$ which we assume in the ${ }^{57}$ Fe layer. (c) The angular dependencies of the Mössbauer reflectivity with the rotated $\pi \rightarrow \sigma$ polarization, calculated for the $1 \mathrm{st}(\mathrm{v}=-5 \mathrm{~mm} / \mathrm{s})$ and 6 th lines $(\mathrm{v}=+5 \mathrm{~mm} / \mathrm{s})$ of the resonant spectrum corresponding to $\mathrm{B}_{\mathrm{hf}}=30 \mathrm{~T}$. The dashed vertical lines mark the angles, for which the spectra in (a) are calculated.

The position of the critical angle is determined by the real part of the susceptibility $\chi$ of the layer (or the refraction index $n=1+\chi / 2$ ). For nuclear resonance scattering, this includes the electronic scattering part and the nuclear resonant contribution. The real part of the nuclear resonant contribution has the opposite signs at two sides of the exact resonance. Therefore, the resonant lines of the narrower sextet with $B_{h f}=28 \mathrm{~T}$ have opposite influences on the refraction index and, accordingly, on the critical angle position for the radiation wavelengths corresponding to the 1st and 6th lines of the broader sextet. Because the maximum of reflectivity for these two lines occurs at slightly different angles, the relative intensities of these two lines are not the same (as they are supposed to be for the absorption case), and they are changed with a small variation of the grazing angle. This provides the observed asymmetry of the spectra depending on the small variation of the grazing angle observed in Figure 8. Note that the studied sample shows even more low-field $\mathrm{B}_{\mathrm{hf}}{ }^{(\mathrm{i})}$ contributions. However, the effect of all smaller fields is, in principle, the same as revealed by the simplest model calculations.

Note that the same changes of the asymmetry of the Mössbauer reflectivity spectra were observed with this particular sample in measurements at the "magnetic" maximum [59]. Although those measurements were performed without the polarization analysis, the "magnetic" maximum is formed basically by the scattering with the rotated $\pi \rightarrow \sigma$ polarization (as shown by the model calculations in Figure 1d,i).

\section{Summary}

We have performed the first experiment on the nuclear resonant reflectivity in the energy domain with the polarization selection, with the two asymmetric $\mathrm{Si}(840)$ reflections from a silicon channel-cut crystal. The experiment has been performed using the synchrotron Mössbauer source, benefiting from the purely linear $\pi$-polarized radiation of this instrument. We have measured the angular dependence of the $\pi \rightarrow \sigma$ reflectivity as well as the Mössbauer $\pi \rightarrow \sigma$ reflectivity spectra at two angles in vicinity of the total external reflection.

We have observed a new effect not reported before. Namely, the nuclear resonance (Mössbauer) $\pi \rightarrow \sigma$ reflectivity in the energy domain is characterized by a peak near critical angle of the total external 
reflection (instead of the Fresnel plateau) similar to that observed for the nuclear resonant reflectivity in the time domain. The effect is explained by the influence of the X-ray standing waves on the nuclear resonant scattering.

Our first experimental realization of the new method shows that, for the polarization analysis in reflectivity experiments, a thorough choice of the right way of polarization selection is needed. The reason is that the reflectivity from cluster-layered films has essentially broadened angular divergence due to a surface roughness and other imperfections. The channel-cut $\mathrm{Si}(840)$ reflection, effectively used in previous polarization experiments in forward scattering or for crystal diffraction $[32,33]$ has angular acceptance that is too small and we lost most of the reflected radiation. For this reason, the quality of the measured Mössbauer $\pi \rightarrow \sigma$ reflectivity spectra was not good enough for quantitative interpretation and our fit just demonstrates the future possibilities. It is clear that other polarization analyzers should be tested (e.g., Be (006) reflection, LiF (622) reflection, Ge (664) reflection, a pyrolytic graphite (222) reflection or some others for $14.4 \mathrm{kev}$ radiation etc.) in order to enlarge the registered signal. Note that in the soft $\mathrm{X}$-ray region a rather unusual way of polarization selection is used (an electron time-of-flight polarization analyzer) [60]. In [61] an annealed silicon crystal was used in order to enlarge the angular acceptance of this monochromator/analyzer. We believe that the right choice of the polarization analyzer allows one to measure Mössbauer $\pi \rightarrow \sigma$ reflectivity with essentially better statistics and to use and demonstrate all theoretically described advantages of the new method of registration.

We have shown (at least by model calculations) that the polarization analysis in Mossbauer spectroscopy simplifies the Mössbauer spectra, restricting the number of lines. It should be helpful for their interpretation in cases of complicated hyperfine interactions (typical for Mössbauer nuclei at a surface, in nano-objects and in thin layers). Moreover, in the reflectivity geometry the spectra lost the dispersive-like features caused by the interference of nuclear and electronic scattering. The enhanced surface sensitivity of the reflectivity signal with rotated $\pi \rightarrow \sigma$ polarization is also predicted, which follows from the "squared-standing-wave" depth dependence of the scattered radiation.

Concluding, we believe that the described advantages of Mössbauer spectroscopy with polarization selection will help to resolve most important scientific cases in surface science, with new physics and high data quality, and will provide us with a more reliable interpretation of the magnetic ordering in multilayer films.

Author Contributions: Conceptualization, M.A.; methodology, A.C., R.R.; investigation, M.A., R.B., T.K., A.C.; software, M.A.; data treatment, R.B., T.K.

Funding: This work was supported by the Russian Foundation of the Basic Research through the Grants No. 15-02-1502 and 16-02-00887-a. R.B. acknowledges a personal grant from the Foundation for the Advancement of Theoretical Physics and Mathematics "BASIS" (grant 18-2-6-22-1).

Acknowledgments: The authors are thankful to Yu. A. Babanov, D. A. Ponomarev and M. A. Milyaev (all IMP UB RAS) for the assigned samples.

Conflicts of Interest: The authors declare no conflict of interest.

\section{References}

1. Schütz, G.; Knülle, V.; Ebert, H. X-ray magnetic circular dichroism and its relation to local magnetic structure. In Resonant Anomalous X-Ray Scattering; Materlik, G., Sparks, C.J., Fischer, K., Eds.; Elsevier Science: New York, NY, USA, 1994.

2. Stöhr, J. X-ray magnetic circular dichroism spectroscopy of transition metal thin films. J. Electron Spectrosc. Relat. Phenom. 1995, 75, 253-272. [CrossRef]

3. Kortright, J.B.; Awschalom, D.D.; Stöhr, J.; Bader, S.D.; Idzerda, Y.U.; Parkin, S.S.P.; Schuller, I.K.; Siegmann, H.-C. Research frontiers in magnetic materials at soft X-ray synchrotron radiation facilities. J. Magn. Magn. Mater. 1999, 207, 7-44. [CrossRef]

4. Van der Laan, G. Applications of soft X-ray magnetic dichroism. J. Phys. Conf. Ser. 2013, 430, 012127. [CrossRef] 
5. Sorg, C.; Scherz, A.; Baberschke, K.; Wende, H.; Wilhelm, F.; Rogalev, A.; Chadov, S.; Minár, J.; Ebert, H. Detailed fine structure of $x$-ray magnetic circular dichroism spectra: Systematics for heavy rare-earth magnets. Phys. Rev. B 2007, 75, 064428. [CrossRef]

6. Sève, L.; Jaouen, N.; Tonnerre, J.M.; Raoux, D.; Bartolomé, F.; Arend, M.; Felsch, W.; Rogalev, A.; Goulon, J.; Gautier, C.; et al. Profile of the induced $5 \mathrm{~d}$ magnetic moments in Ce/Fe and La/Fe multilayers probed by x-ray magnetic-resonant scattering. Phys. Rev. B 1999, 60, 9662-9674. [CrossRef]

7. Ishimatsu, N.; Hashizume, H.; Hamada, S.; Hosoito, N.; Nelson, C.S.; Venkataraman, C.T.; Srajer, G.; Lang, J.C. Magnetic structure of $\mathrm{Fe} / \mathrm{Gd}$ multilayers determined by resonant X-ray magnetic scattering. Phys. Rev. B 1999, 60, 9596-9606. [CrossRef]

8. Blume, M.; Gibbs, D. Polariztion dependence of magnetic x-ray scattering. Phys. Rev. B 1988, 37, $1779-1789$. [CrossRef]

9. Moncton, D.E.; Gibbs, D.; Bohr, J. Magnetic x-ray scattering with synchrotron radiation. Nucl. Instrum. Methods Phys. Res. A 1986, 246, 839-844. [CrossRef]

10. McWhan, D.B.; Vettier, C.; Isaacs, E.D.; Ice, G.E.; Siddons, D.P.; Hastings, J.B.; Peters, C.; Vogt, O. Magnetic x-ray-scattering study of uranium arsenide. Phys. Rev. B 1990, 42, 6007-6017. [CrossRef]

11. Bohr, J. Magnetic x-ray scattering: A new tool for magnetic structure investigations. J. Magn. Magn. Mater. 1990, 83, 530-534. [CrossRef]

12. Langridge, S.; Lander, G.H.; Bernhoeft, N.; Stunault, A.; Vettier, C.; Grübel, G.; Sutter, C.; de Bergevin, F.; Nuttall, W.J.; Stirling, W.G.; et al. Separation of the spin and orbital moments in antiferromagnetic UAs. Phys. Rev. B 1997, 55, 6392-6398. [CrossRef]

13. Sasaki, Y.; Okube, M.; Sasaki, S. Resonant and non-resonant magnetic scatterings with circularly polarized X-rays: Magnetic scattering factor and electron density of gadolinium iron garnet. Acta Cryst. A 2017, 73, 257-270. [CrossRef] [PubMed]

14. Siddons, D.P.; Hart, M.; Amemiya, Y.; Hastings, J.B. X-Ray Optical Activity and the Faraday Effect in Cobalt and Its Compounds. Phys. Rev. Lett. 1990, 64, 1967-1970. [CrossRef] [PubMed]

15. Kortright, J.; Rice, M. Soft X-ray magneto-optic Kerr rotation and element-specific hysteresis measurement. Rev. Sci. Instrum. 1996, 67, 3353. [CrossRef]

16. Collins, S.P. X-ray Faraday rotation and magnetic circular dichroism in an iron-platinum compound. J. Phys. Condens. Matter. 1999, 11, 1159-1175. [CrossRef]

17. Mertins, H.-C.; Schäfers, F.; Gaupp, A.; Le Cann, X.; Gudat, W. Faraday rotation at the $2 p$ edges of Fe, Co, and Ni. Phys. Rev. B 2000, 61, R874-R877. [CrossRef]

18. Kortright, J.B.; Kim, S.-K. Resonant magneto-optical properties of Fe near its $2 p$ levels: Measurement and applications. Phys. Rev. B 2000, 62, 12216-12228. [CrossRef]

19. Mertins, H.-C.; Oppeneer, P.M.; Kuneš, J.; Gaupp, A.; Abramsohn, D.; Schäfers, F. Observation of the X-Ray Magneto-Optical Voigt Effect. Phys. Rev. Lett. 2001, 87, 047401. [CrossRef]

20. Oppeneer, P. Magneto-optical Kerr spectra. Handb. Magn. Mater. 2001, 13, 229-422.

21. Mertins, H.C.; Valencia, S.; Abramsohn, D.; Gaupp, A.; Gudat, W.; Oppeneer, P.M. X-ray Kerr rotation and ellipticity spectra at the 2 p edges of Fe, Co, and Ni. Phys. Rev. B 2004, 69, 064407. [CrossRef]

22. Mertins, H.-C.; Valencia, S.; Gaupp, A.; Gudat, W.; Oppeneer, P.M.; Schneider, C.M. Magneto-optical polarization spectroscopy with soft X-rays. Appl. Phys. A 2005, 80, 1011-1020. [CrossRef]

23. Frauenfelder, H.; Nagle, D.E.; Taylor, R.D.; Cochran, D.R.; Visscher, W.M. Elliptical Polarization of Fe Gamma Rays. Phys. Rev. 1961, 126, 1065-1075. [CrossRef]

24. Blume, M.; Kistner, O.C. Resonant absorption in the presence of Faraday rotation. Phys. Rev. 1968, 171, 417-425. [CrossRef]

25. Housley, R.M.; Grant, R.W.; Gonser, U. Coherence and Polarization Effects in Mössbauer Absorption by Single Crystals. Phys. Rev. 1979, 178, 514-522. [CrossRef]

26. Gerdau, E.; Rüffer, R.; Winkler, H.; Tolksdorf, W.; Klages, C.P.; Hannon, J.P. Nuclear Bragg diffraction of synchrotron radiation in Yttrium Iron Garnet. Phys. Rev. Lett. 1985, 54, 835-838. [CrossRef]

27. Hastings, J.B.; Siddons, D.P.; van Bürck, U.; Hollatz, R.; Bergmann, U. Mössbauer spectroscopy using synchrotron radiation. Phys. Rev. Lett. 1991, 66, 770-773. [CrossRef] [PubMed]

28. Ramachandrah, G.S.; Ramaseshah, S. Crystal Optics. In Handbuch der Physic; Springer: Berlin, Germany, 1961; pp. 1-127. 
29. Smirnov, G.V. General properties of nuclear resonant scattering. Hyperfine Interact. 1999, 123, 31-77. [CrossRef]

30. Siddons, D.P.; Bergmann, U.; Hastings, J.B. Time-dependent polarization in Mössbauer experiments with synchrotron radiation: Suppression of electronic scattering. Phys. Rev. Lett. 1993, 70, 359-362. [CrossRef]

31. Siddons, D.P.; Bergmann, U.; Hastings, J.B. Polarization effects in resonant nuclear scattering. Hyperfine Interact. 1999, 123/124, 681-719. [CrossRef]

32. Toellner, T.S.; Alp, E.E.; Sturhahn, W.; Mooney, T.M.; Zhang, X.; Ando, M.; Yoda, Y.; Kikuta, S. Polarizer/analyzer filter for nuclear resonant scattering of synchrotron radiation. Appl. Phys. Lett. 1995, 67, 1993-1995. [CrossRef]

33. L'abbé, C.; Coussement, R.; Odeurs, J.; Alp, E.E.; Sturhahn, W.; Toellner, T.S.; Johnson, C. Experimental demonstration of time-integrated synchrotron-radiation spectroscopy with crossed polarizer and analyzer. Phys. Rev. B 2000, 61, 4181-4185. [CrossRef]

34. Smirnov, G.V.; van Bürck, U.; Chumakov, A.I.; Baron, A.Q.R.; Rüffer, R. Synchrotron Mössbauer source. Phys. Rev. B 1997, 55, 5811-5815. [CrossRef]

35. Mitsui, T.; Seto, M.; Kikuta, S.; Hirao, N.; Ooishi, Y.; Takei, H.; Kobayashi, Y.; Kitao, S.; Higashitaniguchi, S.; Masuda, R. Generation and Application of Ultrahigh Monochromatic X-ray Using High-Quality ${ }^{57} \mathrm{FeBO}_{3}$ Single Crystal. Jpn. J. Appl. Phys. 2007, 46, 821-825. [CrossRef]

36. Mitsui, T.; Hirao, N.; Ohishi, Y.; Masuda, R.; Nakamura, Y.; Enoki, H.; Sakaki, K.; Seto, M. Development of an energy-domain ${ }^{57} \mathrm{Fe}-\mathrm{Mossbauer}$ spectrometer using synchrotron radiation and its application to ultrahigh-pressure studies with a diamond anvil cell. J. Synchrotron Radiat. 2009, 16, 723-729. [CrossRef] [PubMed]

37. Potapkin, V.; Chumakov, A.I.; Smirnov, G.V.; Celse, J.-P.; Rüffer, R.; McCammon, C.; Dubrovinsky, L. The ${ }^{57}$ Fe Synchrotron Mössbauer Source at the ESRF. J. Synchrotron Radiat. 2012, 19, 559-569. [CrossRef] [PubMed]

38. Andreeva, M.A.; Baulin, R.B.; Chumakov, A.I.; Rüffer, R.; Smirnov, G.V.; Babanov, Y.A.; Devyaterikov, D.I.; Milyaev, M.A.; Ponomarev, D.A.; Romashev, L.N.; et al. Nuclear resonance reflectivity from $\left[{ }^{57} \mathrm{Fe} / \mathrm{Cr}\right]_{30}$ multilayer with Synchrotron Mössbauer Source. J. Synchrotron Radiat. 2018, 25, 473-483. [CrossRef]

39. Mitsui, T.; Imai, Y.; Masuda, R.M.; Seto, M.; Mibu, K. ${ }^{57} \mathrm{Fe}$ polarization-dependent synchrotron Mössbauer spectroscopy using a diamond phase plate and an iron borate nuclear Bragg monochromator. J. Synchrotron Radiat. 2015, 22, 427-435. [CrossRef]

40. Trammell, G.T. Elastic Scattering at Resonance from bound nuclei. Phys. Rev. 1962, 126, 1045-1054. [CrossRef]

41. Andreeva, M.A.; Kuz'min, R.N. Mössbauerovskaya Gamma Optika (in Russian); Moscow Universitet Publ.: Moscow, Russia.

42. Bernstein, S.; Campbell, E.C. Nuclear anomalous dispersion in ${ }^{57} \mathrm{Fe}$ by the method of total reflection. Phys. Rev. 1963, 1625-1633. [CrossRef]

43. Andreeva, M.A.; Rosete, C. Theory of reflection from Mössbauer mirror. Taking account of laminar variation in the parameters of the hyperfine interactions close to the surface. Vestnik Moskovskogo Universiteta Fizika 1986, 41, 57-62.

44. Röhlsberger, R. Nuclear resonant scattering of synchrotron radiation from thin films. Hyperfine Interact. 1999, 123/124, 455-479. [CrossRef]

45. Andreeva, M.; Gupta, A.; Sharma, G.; Kamali, S.; Okada, K.; Yoda, Y. Field induced spin reorientation in $[\mathrm{Fe} / \mathrm{Cr}]_{\mathrm{n}}$ multilayers studied by nuclear resonance reflectivity. Phys. Rev. B 2015, 92, 134403. [CrossRef]

46. Andreeva, M.A.; Lindgren, B.; Panchuck, V. REFTIM. Available online: http://www.esrf.eu/ Instrumentation/software/data-analysis/OurSoftware/REFTIM-1 (accessed on 25 August 2009).

47. Baron, A.Q.R.; Arthur, J.; Ruby, S.L.; Chumakov, A.I.; Smirnov, G.V.; Brown, G.S. Angular dependence of specular resonant nuclear scattering of $x$ rays. Phys. Rev. B 1994, 50, 10354-10357. [CrossRef]

48. Andreeva, M.A.; Lindgren, B. Standing waves and reflectivity from an ultrathin layer. JETP Lett. 2002, 76, 704-706. [CrossRef]

49. Andreeva, M.; Lindgren, B. Nuclear resonant spectroscopy at Bragg reflections from periodic multilayers: Basic effects and applications. Phys. Rev. B 2005, 72, 125422. [CrossRef]

50. Andreeva, M.A.; Baulin, R.A.; Repchenko, Y.L. Standing wave approach in the theory of x-ray magnetic reflectivity. arXiv, 2018; arXiv:1804.05104. 
51. Andreeva, M.A.; Baulin, R.A.; Borisov, M.M.; Gan'shina, E.A.; Kurlyandskaya, G.V.; Mukhamedzhnov, E.K.; Repchenko, Y.L.; Svalov, A.V. Magnetic Dichroism in the Reflectivity of Linearly Polarized Synchrotron Radiation from a $\mathrm{Ti}(10 \mathrm{~nm}) / \mathrm{Gd}_{0.23} \mathrm{Co}_{0.77}(250 \mathrm{~nm}) / \mathrm{Ti}(10 \mathrm{~nm})$ Sample. J. Exp. Theor. Phys. 2018, 125, 802-810. [CrossRef]

52. Henke, B.L. Ultrasoft-X-Ray Reflection, Refraction, and Production of Photoelectrons (100-1000-eV Region). Phys. Rev. A 1972, 6, 94-104. [CrossRef]

53. Bedzyk, M.J.; Bommarito, G.M.; Schildkraut, J.S. X-ray standing waves at a reflecting mirror surface. Phys. Rev. Lett. 1989, 62, 1376-1379. [CrossRef] [PubMed]

54. Chumakov, A.I.; Smirnov, G.V. Mössbauer spectroscopy of conversion electrons: Determining the range of depths that can be analyzed by nondestructive depth profiling. Sov. Phys. JETP 1985, 62, 1044.

55. Andreeva, M.A.; Baulin, R.A.; Chumakov, A.I.; Rüffer, R.; Smirnov, G.V.; Babanov, Y.A.; Devyaterikov, D.I.; Goloborodsky, B.Y.; Ponomarev, D.A.; Romashev, L.N.; et al. Field-temperature evolution of the magnetic state of $[\mathrm{Fe}(1.2 \AA) / \mathrm{Cr}(10.5 \AA)]^{* 30}$ sample by Mössbauer reflectometry with synchrotron radiation. J. Magn. Magn. Mater. 2017, 440, 225-229. [CrossRef]

56. Rüffer, R.; Chumakov, A.I. Nuclear Resonance Beamline at ESRF. Hyperfine Interact. 1996, 97/98, 589-604. [CrossRef]

57. Chumakov, A.I.; Sergeev, I.; Celse, J.-P.; Rüffer, R.; Lesourd, M.; Zhang, L.; Sanchez del Rio, M. Performance of a silicon monochromator under high heat load. J. Synchrotron Radiat. 2014, 21, 315-324. [CrossRef] [PubMed]

58. Ragulskaya, A.V.; Andreeva, M.A.; Rogachev, M.A.; Yakunin, S.N. The investigation of [Fe/Cr] multilayer by GISAXS. Superlattices Microstruct. 2019, 125, 16-25. [CrossRef]

59. Andreeva, M.A.; Chumakov, A.I.; Smirnov, G.V.; Babanov, Y.A.; Devyaterikov, D.I.; Goloborodsky, B.Y.; Ponomarev, D.A.; Romashev, L.N.; Ustinov, V.V.; Rüffer, R. Striking anomalies in shape of the Mössbauer spectra measured near "magnetic" Bragg reflection from $[\mathrm{Fe} / \mathrm{Cr}]$ multilayer. Hyperfine Interact. 2016, 237, 1-9. [CrossRef]

60. Müller, L.; Hartmann, G.; Schleitzer, S.; Berntsen, M.H.; Walther, M.; Rysov, R.; Roseker, W.; Scholz, F.; Seltmann, J.; Glaser, L.; et al. Note: Soft X-ray transmission polarizer based on ferromagnetic thin films. Rev. Sci. Instrum. 2018, 89, 036103. [CrossRef] [PubMed]

61. Schneider, J.R.; Nagasawa, H.; Berman, L.E.; Hastings, J.B.; Siddons, D.P.; Zulehner, W. Test of annealed Czochralski grown silicon crystals as X-ray diffraction elements with $145 \mathrm{keV}$ synchrotron radiation. Nucl. Instrum. Methods 1989, A276, 636-642. [CrossRef] 